\title{
Unresectable Fibrolamellar Carcinoma
}

National Cancer Institute

\section{Source}

National Cancer Institute. Unresectable Fibrolamellar Carcinoma. NCI Thesaurus. Code C162602.

A fibrolamellar carcinoma that is not amenable to surgical resection. 